\title{
Specialized transduction: an efficient method for generating marked and unmarked targeted gene disruptions in Mycobacterium tuberculosis, M. bovis BCG and M. smegmatis
}

\author{
Stoyan Bardarov, ${ }^{1}$ Svetoslav Bardarov, $\mathrm{Jr}^{1}{ }^{1}$ Martin S. Pavelka, $\mathrm{Jr}^{4}{ }^{4}$ \\ Vasan Sambandamurthy, ${ }^{1,2}$ Michelle Larsen, ${ }^{1,2}$ JoAnn Tufariello, ${ }^{1,3}$ \\ John Chan, ${ }^{1,3}$ Graham Hatfull ${ }^{5}$ and William R. Jacobs, Jr ${ }^{1,2}$
}
1,2 Dept of Microbiology and Immunology ${ }^{1}$ and Howard Hughes Medical Institute ${ }^{2}$, Albert Einstein College of Medicine, Bronx, NY 10461, USA
3 Division of Infectious Diseases, Department of Medicine, Montefiore Medical Center, Bronx, NY, USA
4 Department of Microbiology and Immunology, University of Rochester Medical Center, 601 Elmwood Ave., Rochester, NY 14642, USA
5 Department of Biological Sciences, Bacteriophage Institute, University of Pittsburgh, 365A Crawford Hall, Pittsburgh, PA 15260, USA

\author{
Author for correspondence: William R. Jacobs, Jr. Tel: +1 718430 2888. Fax: +1 7184308844. \\ e-mail: jacobsw@hhmi.org
}

The authors have developed a simple and highly efficient system for generating allelic exchanges in both fast- and slow-growing mycobacteria. In this procedure a gene of interest, disrupted by a selectable marker, is cloned into a conditionally replicating (temperature-sensitive) shuttle phasmid to generate a specialized transducing mycobacteriophage. The temperaturesensitive mutations in the mycobacteriophage genome permit replication at the permissive temperature of $30^{\circ} \mathrm{C}$ but prevent replication at the nonpermissive temperature of $37^{\circ} \mathrm{C}$. Transduction at a non-permissive temperature results in highly efficient delivery of the recombination substrate to virtually all cells in the recipient population. The deletion mutations in the targeted genes are marked with antibiotic-resistance genes that are flanked by $\gamma \delta$-res (resolvase recognition target) sites. The transductants which have undergone a homologous recombination event can be conveniently selected on antibiotic-containing media. To demonstrate the utility of this genetic system seven different targeted gene disruptions were generated in three substrains of Mycobacterium bovis BCG, three strains of Mycobacterium tuberculosis, and Mycobacterium smegmatis. Mutants in the lysA, nadBC, panC, panCD, leuCD, Rv3291C and Rv0867c genes or operons were isolated as antibiotic-resistant (and in some cases auxotrophic) transductants. Using a plasmid encoding the $\gamma \delta$-resolvase (tnpR), the resistance genes could be removed, generating unmarked deletion mutations. It is concluded from the high frequency of allelic exchange events observed in this study that specialized transduction is a very efficient technique for genetic manipulation of mycobacteria and is a method of choice for constructing isogenic strains of M. tuberculosis, BCG or M. smegmatis which differ by defined mutations.

Keywords: mycobacteriophage, homologous recombination, allelic exchange

\section{INTRODUCTION}

Tuberculosis continues to be a major public health problem. With the rapid increase of drug-resistant strains of Mycobacterium tuberculosis, the need for an improved vaccine to combat the disease has become an international priority (see http://www.who.int/inf-

Abbreviations: AES, allelic exchange substrate; MCS, multiple cloning site. fs/en/fact104.html). The determination of the genome sequence of two strains of $M$. tuberculosis (Cole et al., 1998; Fleischmann, 2002), available in two public domains (http://genolist.pasteur.fr/TubercuList and http://www.tigr.org), promises to accelerate the increase in our knowledge concerning the fundamental biology of these organisms. However, an efficient methodology to generate defined mutations is urgently needed for the rapid development of the functional genomics of the clinically important pathogenic myco- 
bacteria in order to understand the genetic basis for their tropism, virulence and persistence in the host.

Mutant isolation and gene transfer strategies have been successfully used for the fast-growing mycobacteria such as Mycobacterium smegmatis (Boshoff \& Mizrahi, 2000; Braunstein et al., 2001; Frischkorn et al., 1998; Knipfer et al., 1997; Pavelka \& Jacobs, 1999). However, in the slow-growing mycobacterial species the construction of genetically defined isogenic strains containing single or multiple mutations has been notoriously difficult. This is primarily due to the high frequency of illegitimate recombination in these organisms (Aldovini et al., 1993; Kalpana et al., 1991) as well as their intrinsic tendency to grow in aggregates (clumps), which makes the isolation of individual clones problematic. The difficulties encountered in early attempts at allelic exchange led to the general conclusion that homologous recombination in the slow-growing mycobacteria is inefficient (McFadden, 1996).

Several groups have reported successful gene disruptions in M. tuberculosis and Mycobacterium bovis BCG using short (Azad et al., 1996; Kalpana et al., 1991; Reyrat et al., 1995) or long linear DNA fragments (Balasubramanian et al., 1996) as allelic exchange substrates (AESs) for homologous recombination. A 'suicide' vector approach, using recombinant plasmids unable to replicate in mycobacteria, was extensively used to achieve allelic exchange in both fast- and slow-growing mycobacteria (Berthet et al., 1998; Fitzmaurice \& Kolattukudy, 1998; Knipfer et al., 1997; Parish et al., 1999; Pavelka \& Jacobs, 1996; Pelicic et al., 1996a, b, 1997; Sander et al., 1995). A two-step selection method using selectable and counterselectable markers, positioned on either replicating or non-replicating plasmids, has been also successfully used in M. smegmatis (Knipfer et al., 1997; Pelicic et al., 1996a), M. bovis BCG and M. tuberculosis (Hinds et al., 1999; Parish et al., 1999; Parish \& Stoker, 2000; Pavelka \& Jacobs, 1999). Unfortunately, the suicide vector approach (using a nontemperature-sensitive plasmid) is dependent upon the delivery of the AES by electroporation. Because homologous recombination frequencies are very close to the efficiency at which plasmids can be electroporated into slow-growing mycobacteria, the suicide vector approach is limited to those cases where high transformation efficiencies can be obtained. It has been proposed that this electroporation limitation, not inefficient homologous recombination, is the reason for earlier difficulties encountered in allelic exchange experiments in slowgrowing mycobacteria (Pavelka \& Jacobs, 1999). The use of conditionally replicating temperature-sensitive plasmid replicons as delivery vectors has greatly improved reproducibility of allelic exchange in the slowgrowing mycobacteria (Pelicic et al., 1997), although growth of the cultures at low temperature is required, which may not be expedient.

General transduction, which is based on the natural genetic exchange of DNA information, is an alternative strategy to efficiently introduce homologous DNA into the recipient cells by bacteriophages (Lenox,
1955; Masters, 1996; Zinder \& Lederberg, 1952). Transductional transfer of AESs has greatly facilitated the generation of specific mutations and the functional analysis of the genomes of Escherichia coli and Salmonella. However, while the transfer of DNA by genetic transduction has been reported for $M$. smegmatis (Sundar Raj \& Ramakrishnan, 1970), it has not been reported yet for the slow-growing mycobacteria such as M. bovis BCG and M. tuberculosis. The ability to transfer DNA to slow-growing mycobacteria by a highly efficient, phage-based method would overcome the electroporation limitations described above for the plasmid transformation methods of allelic exchange.

This report describes a novel genetic method for mycobacteria for the generation of targeted deletion mutations by allelic exchange using in vitro-generated specialized transducing mycobacteriophages. The utility and reproducibility of this method have been demonstrated by the construction of seven isogenic auxotrophic mutant strains of $M$. smegmatis, three substrains of M. bovis BCG and three strains of M. tuberculosis. The effectiveness of this method has also been shown by several other researchers, who successfully engineered numerous targeted gene disruptions in M. tuberculosis (Glickman et al., 2000; Raman et al., 2001; Sirakova et al., 2001; Steyn et al., 2002), using the reagents described or their derivatives, prior to submission of this article. We also demonstrate the efficient elimination of the resistance gene (unmarking the mutation) by using a plasmid expressing the $\gamma \delta$-TnpR site-specific resolvase, which acts on the directly repeated res sites flanking the resistance gene. Based on the observed high frequency of allelic exchange and the subsequent efficient removal of the marker gene we conclude that this is a powerful genetic method for engineering targeted marked and unmarked mutations in various mycobacterial species, particularly in the slow-growing pathogenic mycobacteria and those in which it is difficult to achieve efficient plasmid transformation.

\section{METHODS}

Bacterial strains, media and growth conditions. The E. coli strains were grown in Luria-Bertani (LB) broth or on LB agar (Difco) for the amplification of recombinant clones, plasmid isolation and transformation, and in TYM broth $(1 \%$ tryptone, $0.5 \%$ yeast extract, $0.5 \% \mathrm{NaCl}, 0 \cdot 2 \%$ maltose) for transduction with $\lambda$-packaged cosmids. For the preparation of electrocompetent cells M. smegmatis $\mathrm{mc}^{2} 155$ was grown in LB containing $0.5 \%$ (w/v) Tween 80 (LBT) (Pavelka \& Jacobs, 1999). When required, the following antibiotics were used at the specified concentrations: carbenicillin $\left(50 \mu \mathrm{g} \mathrm{ml}^{-1}\right)$, kanamycin $\left(25 \mu \mathrm{g} \mathrm{ml}^{-1}\right)$ and hygromycin $\mathrm{B}\left(50 \mu \mathrm{g} \mathrm{ml}^{-1}\right.$ for E. coli and $150 \mu \mathrm{g} \mathrm{ml}^{-1}$ for $M$. smegmatis). Mycobacterial strains (except for M. smegmatis) were grown in basal Middlebrook $7 \mathrm{H} 9$ (Difco) supplemented with $1 \times$ ADS $[0.5 \%$ bovine serum albumin, fraction $\mathrm{V}$ (Boehringer Manheim), $0.2 \%$ glucose and $0.085 \% \mathrm{NaCl}], \quad 0 \cdot 2 \%$ glycerol and $0 \cdot 1 \%$ Tween 80 (7H9ADSTW broth) (Jacobs et al., 1991). Complete medium consisted of 7H9ADSTW supplemented with individual Lamino acids at a final concentration of $40 \mu \mathrm{g} \mathrm{ml}^{-1}$. Pantothenic acid and nicotinamide (Sigma) were used at $20 \mu \mathrm{g} \mathrm{ml}^{-1}$. For transduction experiments, cultures of the M. bovis BCG or $M$. 
(a)

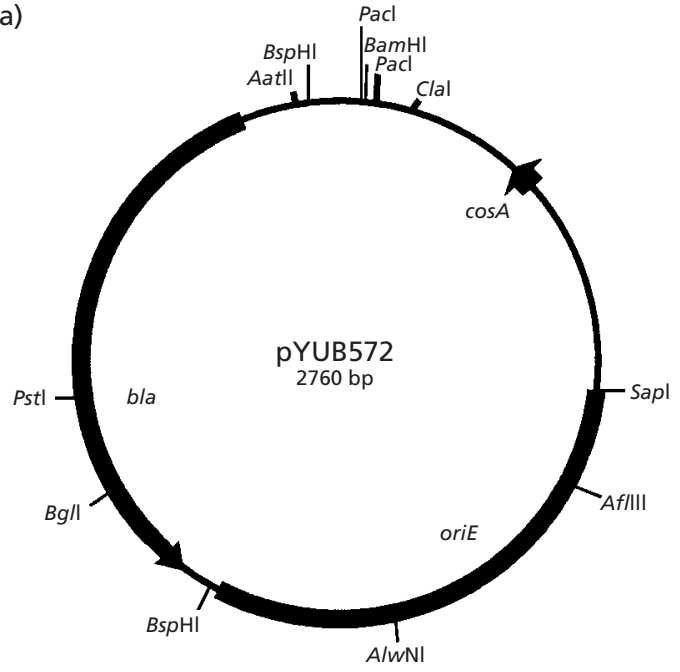

(b)

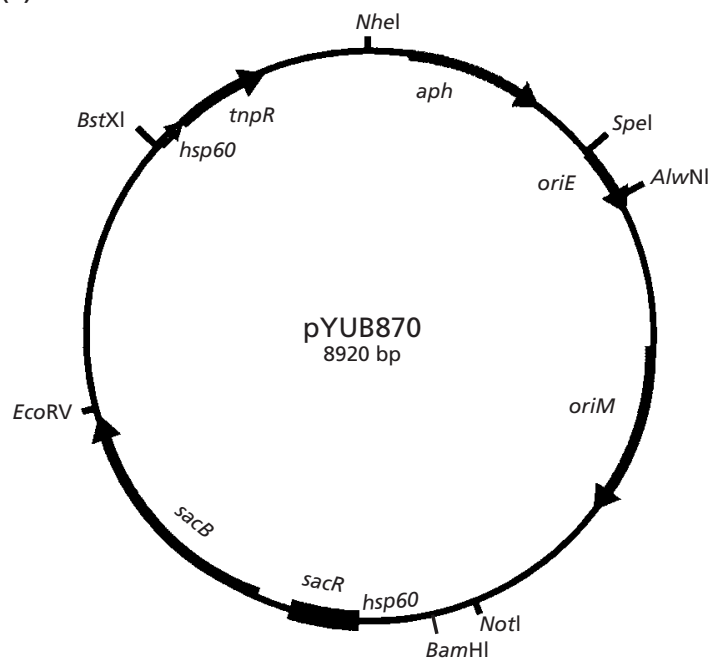

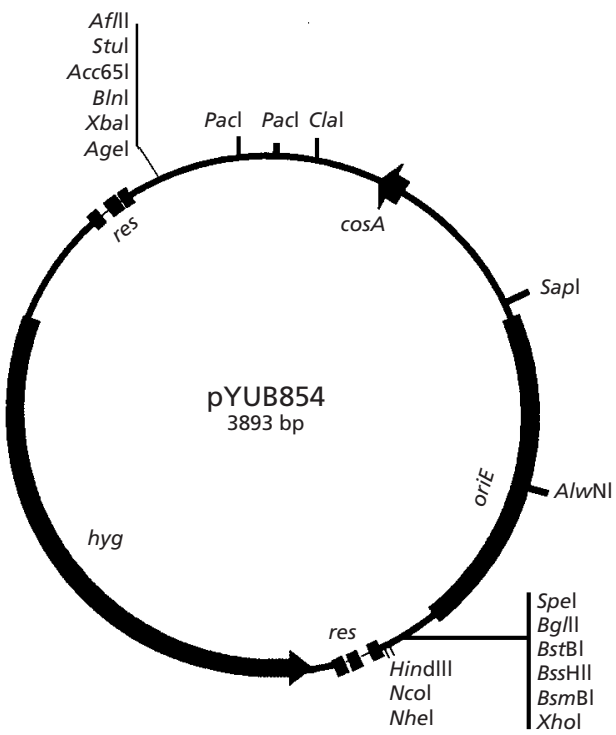

Fig. 1. Maps of the cosmids pYUB572 and pYUB854, and the helper plasmid pYUB870. (a) Cosmid vectors pYUB572 and pYUB854. Common features are the presence of origin of replication for $E$. coli (oriE), a single $\lambda$-cos site, and $\mathrm{PaCl}$ sites for efficient introduction of the cosmid into the mycobacteriophage shuttle phasmids. The res-hyg-res gene cassette in the cosmid pYUB854 is flanked by the $\gamma \delta$-res sites for subsequent removal of the selectable marker gene and by convenient MCSs for directional cloning of the flanking homologous recombination substrates. (b) The helper plasmid pYUB870 for transient expression of the $\gamma \delta$-tnp $R$ gene. The plasmid has the aph gene conferring resistance to kanamycin, $\mathrm{P}_{h s p 60}-$ SacRB and $\mathrm{P}_{\text {hsp60 }}$-tnpR. tuberculosis strains were prepared by inoculating $10 \mathrm{ml}$ 7H9ADSTW broth with $1 \mathrm{ml}$ frozen stock into $30 \mathrm{ml}$ plastic culture bottles and incubated at $37^{\circ} \mathrm{C}$ in an incubator-shaker. For propagation of mycobacteriophages in M. smegmatis $\mathrm{mc}^{2} 155$, $7 \mathrm{H} 9$ basal medium supplemented with $0 \cdot 4 \%$ glycerol and $2 \mathrm{mM} \mathrm{CaCl}_{2}$ (7H9-Gly-CaCl${ }_{2}$ ) was used as a bottom agar, and $0.6 \%$ agar in water supplemented with $2 \mathrm{mM} \mathrm{CaCl}_{2}$ as a top agar. High-titre mycobacteriophage lysates were prepared from phage propagated in M. smegmatis grown on 7H9-Gly$\mathrm{CaCl}_{2}$ solid medium at $30^{\circ} \mathrm{C}$. Phage lysates were stored in MP buffer $(50 \mathrm{mM}$ Tris/HCl pH 7.6, $150 \mathrm{mM} \mathrm{NaCl}, 10 \mathrm{mM}$ $\mathrm{MgCl}_{2}$ and $2 \mathrm{mM} \mathrm{CaCl}$ ). Adsorption of the phages was performed for $30 \mathrm{~min}$ at $37^{\circ} \mathrm{C}$ for M. smegmatis and for $3 \mathrm{~h}$ at $37^{\circ} \mathrm{C}$ for M. tuberculosis. Outgrowth of the infected cells was performed by transferring the phage/cell mixture to a roller bottle containing $50 \mathrm{ml}$ complete $7 \mathrm{H} 9 \mathrm{ADSTW}$ pre-warmed to $37^{\circ} \mathrm{C}$.

DNA manipulations. DNA manipulations were done essentially as described by Sambrook et al. (1989). High-molecularmass chromosomal DNA from the mycobacterial strains was purified by the CTAB method as described previously (Balasubramanian et al., 1996). Phage DNA from mycobacteriophages was purified as previously described (Jacobs et al.,
1991) with slight modifications. High-titre phage lysates $(30 \mathrm{ml})$ were layered onto a $5 \mathrm{ml}$ cushion of $50 \%$ glycerol in MP buffer in a $35 \mathrm{ml}$ nitrocellulose centrifuge tube (Beckman) and centrifuged at 25000 r.p.m. for $2 \mathrm{~h}$ in an SW27 rotor at $15^{\circ} \mathrm{C}$. Phage pellets were resuspended in $500 \mu \mathrm{l} \mathrm{MP}$ buffer to which $25 \mu \mathrm{l} \quad(0.05$ vol. $)$ of STEP lysis solution (0.4 $\mathrm{M} \mathrm{Na}_{2}$ EDTA $1 \%$ SDS, $50 \mathrm{mM}$ Tris $/ \mathrm{HCl} \mathrm{pH} 8 \cdot 0,200 \mu \mathrm{g}$ Proteinase $\mathrm{K} \mathrm{ml}^{-1}$ ) was added and the suspension was incubated for $30 \mathrm{~min}$ at $56^{\circ} \mathrm{C}$. Following extraction with phenol/ chloroform $(1: 1)$ and chloroform/isoamyl alcohol $(24: 1)$, phage DNA was precipitated by the addition of 2 vols $100 \%$ ethanol, washed in $70 \%$ ethanol, air-dried and resuspended at a concentration of approximately $200 \mu \mathrm{g} \mathrm{ml}^{-1}$ in TE buffer (10 mM Tris/ $\mathrm{HCl} \mathrm{pH} \mathrm{8 \cdot 0,1} \mathrm{mM} \mathrm{Na}_{2}$ EDTA).

Construction of the recombinant cosmids containing allelic exchange substrates (AESs). Cosmid vector pYUB572 is a derivative of pYUB328 (Balasubramanian et al., 1996), in which $2 \cdot 2 \mathrm{~kb}$ fragment containing one of the $\lambda$-cos sites was removed by cleavage with MunI and Csp45I. Cosmid pYUB854 is a derivative of pYUB572, in which the bla gene was removed by digestion with $B s p \mathrm{HI}$ and replaced with a res-hyg-res gene cassette flanked by multiple cloning sites (MCSs). Plasmid pYUB870 is a derivative of pMV261 (Stover 
et al., 1991) in which the $\gamma \delta$-resolvase gene $(m p R)$ from transposon Tn1000 was cloned under the control of the mycobacterial $h s p 60$ promoter. It also contains the $s a c B$ gene, which provides a negative selection for the loss of the helper plasmid when $\operatorname{Kan}^{\mathrm{R}}$ cultures are plated on sucrose-containing media (Fig. 1). The phasmid phAE87 is a derivative of the conditionally replicating mycobacteriophage $\mathrm{PH} 101(t s)$ (Bardarov et al., 1997). The phasmid phAE159, which permits a larger cloning capacity, is a derivative of phAE87 (J. I. Kriakov \& W. R. Jacobs, Jr, unpublished results). The plasmid pYUB619 (Pavelka \& Jacobs, 1999) was used as a source of the M. smegmatis $\Delta l y s A 4:$ :res-hyg-res deletion allele. A $4130 \mathrm{bp}$ BamHI-NotI fragment was cloned into BspHI-digested pYUB572 by blunt-end ligation to generate two cosmids, pYUB804 and pYUB805, which differ in the orientation of the $\Delta l y s A 4::$ res-hyg-res with respect to the BamHI site in the cosmid. Plasmid pYUB665 (Pavelka \& Jacobs, 1999) was used as a source of the M. tuberculosis $\Delta l y s A 5::$ res-aph-res gene. A $4198 \mathrm{bp} \mathrm{BclI-AscI} \mathrm{fragment} \mathrm{from}$ pYUB665, containing the $\Delta l y s A 5:$ :res-hyg-res gene flanked by $\sim 1 \mathrm{~kb}$ of DNA sequence on each side, was cloned by bluntend ligation into BspHI-digested cosmid pYUB572, to generate pYUB586. To generate the panC deletion mutation in $M$. smegmatis, the sequence database (http://www.tigr.org) was utilized to generate two primer pairs, which were employed to amplify the upstream LEFT and the downstream RIGHT arms flanking the panC gene. Flanking arms were cloned directionally into cosmid pYUB854 to generate the recombinant cosmid pYUB2500. PCR amplification of the entire operons using single primer pairs was the method used to generate the leuCD and nadBC AESs. PCR products were cloned into pBluescript KSII cloning vector. Deletions in the operons were generated with the appropriate restriction endonuclease and then marked with res-aph-res $(\Delta l e u C D)$ or res-byg-res $(\Delta n a d B C)$. The AESs thus generated were then cloned by replacing the bla gene of the cosmid pYUB572. PCR amplification of the upstream and the downstream flanking DNA sequences using the $M$. tuberculosis genome sequence database (http://genolist.pasteur.fr/TubercuList) was used to generate the homologous AESs for the generation of deletion mutations in the panCD, Rv0867c and Rv3291c genes. After sequencing, the resulting DNA fragments were cloned directionally into cosmid vector pYUB854 to generate deletion mutants marked with the res-hyg-res gene cassette.

Construction of specialized transducing mycobacteriophages. Concatamers of phAE87 were prepared by selfligation of purified phage DNA, which was then digested with PacI. To generate each specialized transducing phage, the PacI-digested recombinant cosmid carrying the appropriate AES was used to replace the pYUB328 cosmid in phAE87 or phAEB159 in an in vitro $\lambda$-packaging reaction (GIGAPackII, Stratagene). After transducing E. coli HB101 and plating the transductants on selective media containing kanamycin or hygromycin, phasmid DNA was prepared from the pooled antibiotic-resistant transductants and electroporated into $M$. smegmatis $\mathrm{mc}^{2} 155$. All transducing phages were plaquepurified and tested to confirm the temperature-sensitive phenotype. The correct structure of the AES in the specialized transducing phage was confirmed by PCR with locus-specific primers and by Southern blot analysis.

Transduction protocol. M. smegmatis $\mathrm{mc}^{2} 155$ was grown in LBT to an $\mathrm{OD}_{600}$ of $1 \cdot 0\left(\sim 6 \times 10^{8}\right.$ c.f.u. $\left.\mathrm{ml}^{-1}\right)$. M. bovis BCG strains and M. tuberculosis were grown in 7H9ADSTW to an $\mathrm{OD}_{600}$ of $\sim 0 \cdot 8-1 \cdot 0$. Ten millilitres of the culture was centrifuged and resuspended in $10 \mathrm{ml}$ washing medium (7H9ADS without Tween 80) and incubated as a standing culture at $37^{\circ} \mathrm{C}$ for $24 \mathrm{~h}$. This incubation was included to remove traces of the Tween 80 detergent, which can inhibit phage infection. After this incubation period the cells were again centrifuged and resuspended in $1.0 \mathrm{ml}$ 7H9ADS broth without Tween 80 , pre-warmed at $37^{\circ} \mathrm{C}$, and mixed with specialized transducing phage at an m.o.i. of 10 . The cell/phage mixture was incubated at the non-permissive temperature $\left(37^{\circ} \mathrm{C}\right)$ for $30 \mathrm{~min}$ (M. smegmatis) or $3 \mathrm{~h}$ (BCG and $M$. tuberculosis), after which the mixture was inoculated into $50 \mathrm{ml} \mathrm{LBT} \mathrm{(M.} \mathrm{smegmatis)} \mathrm{or} \mathrm{complete} \mathrm{7H9ADSTW}$ (BCG and M. tuberculosis) pre-warmed at $37^{\circ} \mathrm{C}$. Outgrowth of the cultures was performed for $30 \mathrm{~min}$ (M. smegmatis) or $24 \mathrm{~h}$ (BCG and M. tuberculosis) at $37^{\circ} \mathrm{C}$. Cells were then pelleted by centrifugation, resuspended in $1 \mathrm{ml}$ PBS-TW $(0 \cdot 1 \%$ Tween 80 in phosphate-buffered saline) and plated on 7H9ADSTW complete medium containing kanamycin $\left(25 \mu \mathrm{g} \mathrm{ml}^{-1}\right)$ or hygromycin $\left(150 \mu \mathrm{g} \mathrm{ml}^{-1}\right.$ for $M$. smegmatis and $75 \mu \mathrm{g} \mathrm{ml}^{-1}$ for M. tuberculosis). Auxotrophic analysis was performed by plating the transductants on complete medium as well as on minimal medium. Transduction frequencies were calculated by dividing the number of $\mathrm{Hyg}^{\mathrm{R}}$ or $\mathrm{Kan}^{\mathrm{R}}$ colonies obtained minus the number of spontaneous drug-resistant colonies from control cells receiving no phage by the total number of viable cells. The frequency of allelic exchange was calculated as the percentage of auxotrophs in the population of antibiotic-resistant transductants.

PCR analysis and Southern blotting. PCR amplification was performed with AmpliTaq polymerase (Perkin-Elmer) under standard conditions. Primer concentrations and cycling conditions were adjusted depending on the size of the amplified product. All PCR reactions were performed in a Perkin-Elmer 9600 thermal cycler. Southern blotting was done by the alkalidenaturing procedure. DNA was transferred to HyBond-N+ membrane (Amersham) by the capillary method. Hybridization and detection were done with a chemiluminescent detection system (ECL, Amersham) as recommended by the manufacturer, In some cases probes were labelled with $[\alpha-$ ${ }^{32} \mathrm{P}$ ]dCTP using Ready-To-Go DNA Labelling Beads (Amersham Pharmacia Biotech).

\section{RESULTS}

\section{Specialized transduction methodology}

In this study the primary goal was to develop a highly efficient system for the delivery of homologous DNA substrates in order to facilitate the generation of targeted gene disruptions in a wide array of mycobacterial species. Previous studies have shown that conditionally replicating shuttle phasmids are an efficient system for the generation of large libraries of independent transposon mutants in mycobacteria, as they provide the means to efficiently deliver transposon constructs to virtually every cell in the bacterial population (Bardarov et al., 1997; Cox et al., 1999). We reasoned that this recombinant DNA transfer system could be modified into a system for the generation of targeted deletion mutations in mycobacteria by allelic exchange, using in vitro-generated specialized transducing mycobacteriophages.

The specialized transducing mycobacteriophages consist of two basic components: a cosmid vector containing the AES and a conditionally replicating shuttle phasmid vector, which is a derivative of the broad-host-range 
Step 1: Construction of AES

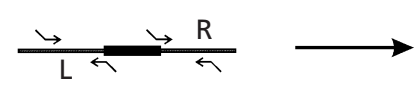

Step 2: Incorporation of AES into shuttle

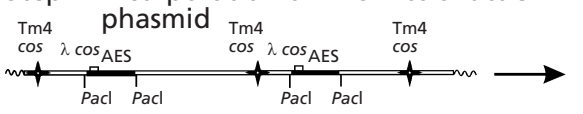

Package in vitro into $\lambda$ heads
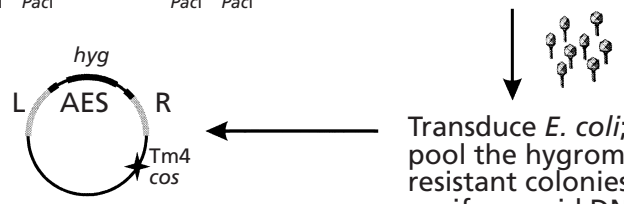

Transduce E. coli; pool the hygromycin resistant colonies and purify cosmid DNA

Step 3: Conversion of the cosmid into mycobacteriophagepackaged shuttle phasmid

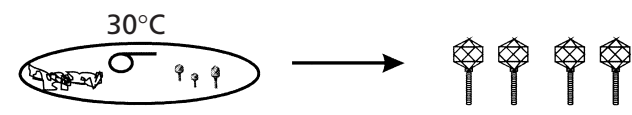

Step 4: Specialized transduction

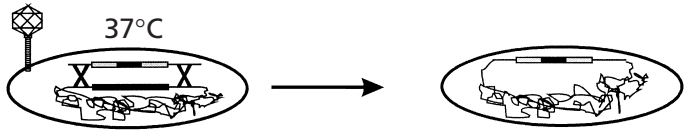

$\Delta y f g::$ res::hyg::res

Step 5: Unmarking the mutation in $\Delta y f g$

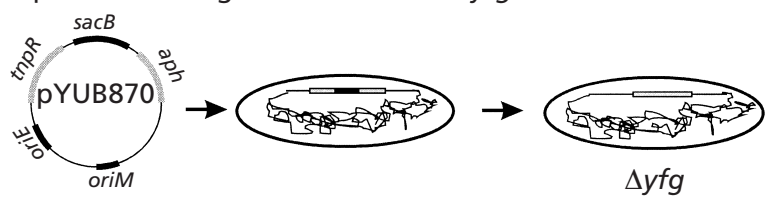

Fig. 2. Schematic of one-step gene replacement in mycobacteria using in vitro-constructed specialized transducing phages.

TM4 phage (PH101ts). Specialized transduction involves five basic steps as outlined in Fig. 2: (1) construction of an AES in a PacI-containing E. coli cosmid; (2) cloning of the recombinant cosmid into the conditionally replicating shuttle phasmid; (3) transfection of $M$. smegmatis at the permissive temperature of $30^{\circ} \mathrm{C}$, to generate mycobacteriophage-packaged shuttle phasmids; (4) phage infection (transduction) of the mycobacteria at the non-permissive temperature of $37^{\circ} \mathrm{C}$; and (5) unmarking the deletion mutation by transient expression of $t n p R$.

(1) Construction of the AES in a Pacl-containing E. coli cosmid. To make a targeted gene disruption of your favourite gene ( $y f g)$, it is necessary to generate an AES that consists of a selectable marker gene and the regions of DNA flanking $y f g$. Such a construct is simply made by using either the cosmid pYUB572 or the cosmid pYUB854, which have several features in common: (a) an origin of replication for E. coli (oriE); (b) a single $\lambda$ cos site allowing in vitro packaging into phage $\lambda$ heads; (c) a mycobacterial selectable marker gene such as $a p h$ or hyg flanked by $\gamma \delta$-res sites for subsequent removal of the selectable marker gene; (d) MCSs flanking the selectable marker gene for directional cloning of the flanking homologous recombination substrates; and (e) a unique PacI site for the efficient introduction of the cosmid into the mycobacteriophage shuttle phasmid.

The AES can be generated in the cosmid pYUB572 by traditional cloning methods, where a DNA fragment containing $y f g$, with $700-1000 \mathrm{bp}$ of upstream and downstream flanking DNA sequence, is amplified by PCR and cloned into any plasmid vector of choice. The desired deletion mutation in $y f g$ is then generated by digestion with the chosen restriction enzyme and marked by insertion of the reporter gene. The marked homologous DNA substrate is then cloned into the cosmid vector pYUB572, by replacing the bla gene and plating on appropriate selective media. The cosmid cloning vector pYUB854, depicted in Fig. 1 and in Fig. 2 (Step 1), provides an alternative strategy for the engineering of AES. The LEFT and RIGHT flanking DNA arms (700-1000 bp long) are generated by PCR using two sets of primers. The region between the LEFT 3' (reverse) and the RIGHT 5' (forward) primers is the portion of the gene which is deleted. To help directional cloning of the DNA arms, the primer pairs may be engineered to contain restriction endonuclease sites corresponding to those found in the MCSs flanking the hygromycinresistance gene cassette. Once this cloning is complete, the LEFT and RIGHT flanking DNA arms will be in their original genome orientation separated by the res-byg-res cassette. The res-byg-res cassette contains the DNA-binding sites (res sites) for the site-specific resolvase $\gamma \delta$-TnpR (Hatfull, 1988; Reed, 1981). When the resolvase gene $(\operatorname{tn} p R)$ is provided by a plasmid and transiently expressed in the mutant host strain, the sitespecific recombination between the res sites results in unmarking of the deletion by precise excision of the hyg gene cassette.

(2) Introduction of the recombinant cosmid into the conditionally replicating TM4 shuttle phasmid. The second step involves cloning of the recombinant cosmid, containing the AES, into the conditionally replicating shuttle phasmid vector phAE87 or phAE159. Shuttle phasmid vectors are useful in that they allow all of the DNA manipulations to be performed in E. coli. However, there is an upper limit to the size of the allelic exchange construct that can be cloned into the mycobacteriophage genome. This limit is set by the size of DNA that can be packaged into $\lambda$ phage heads (about $50 \mathrm{~kb}$ ) for the in vitro packaging and E. coli transduction step, and also by the size of the DNA that can be packaged into TM4 phage heads when transfected into $M$. smegmatis. The shuttle phasmid phAE87 contains a deletion of about $300 \mathrm{bp}$ and is able to accommodate a maximum of $6.0 \mathrm{~kb}$ of exogenous DNA (cosmid vector size included). Any larger-sized phage molecules are very unstable in E. coli in their cosmid form or as mycobacteriophages in $M$. smegmatis. The shuttle phasmid phAE 159 contains a deletion of $5.6 \mathrm{~kb}$ and thus is able to accommodate larger DNA inserts (J. I. Kriakov \& W. R. Jacobs, Jr, unpublished results). 
Table 1. Mutant alleles in $M$. bovis BCG, $M$. tuberculosis and $M$. smegmatis generated by specialized transduction

\begin{tabular}{|c|c|c|c|}
\hline Strain & $\begin{array}{c}\text { Transducing } \\
\text { phage }\end{array}$ & Genotype & $\begin{array}{c}\text { No. of allelic exchanges/ } \\
\text { no. of } \mathrm{Kan}^{\mathrm{R}} \text { or } \mathrm{Hyg}^{\mathrm{R}} \\
\text { transductants* }\end{array}$ \\
\hline \multicolumn{4}{|l|}{ BCG } \\
\hline \multicolumn{4}{|l|}{ Pasteur } \\
\hline $\mathrm{mc}^{2} 1507$ & phAE128 & $\Delta l y s A 5::$ res-aph-res & $168 / 495$ \\
\hline $\mathrm{mc}^{2} 1510$ & phAE134 & $\Delta l e u C D:$ :res-aph-res & $60 / 96$ \\
\hline $\mathrm{mc}^{2} 1511$ & phAE152 & $\Delta$ nadBC::res-byg-res & $4 / 4$ \\
\hline $\mathrm{mc}^{2} 6000$ & phAE187 & $\Delta$ panCD::res-byg-res & $8 / 8$ \\
\hline $\mathrm{mc}^{2} 1512$ & phAE152 & $\Delta l y s A 5, \Delta n a d B C::$ res-aph-res & $4 / 4$ \\
\hline \multicolumn{4}{|l|}{ Copenhagen } \\
\hline $\mathrm{mc}^{2} 1508$ & phAE128 & $\Delta l y s A 5::$ res-aph-res & $125 / 432$ \\
\hline $\mathrm{mc}^{2} 1511$ & phAE134 & $\Delta l e u C D:$ :res-aph-res & $46 / 96$ \\
\hline $\mathrm{mc}^{2} 1513$ & phAE153 & $\Delta n a d B C:$ :res-byg-res & $3 / 3$ \\
\hline \multicolumn{4}{|l|}{ Moreau } \\
\hline $\mathrm{mc}^{2} 1509$ & phAE128 & $\Delta l y s A 5::$ res-aph-res & $13 / 230$ \\
\hline $\mathrm{mc}^{2} 1512$ & phAE134 & $\Delta l e u C D:$ :res-aph-res & $93 / 96$ \\
\hline $\mathrm{mc}^{2} 1514$ & phAE152 & $\Delta n a d B C::$ res-hyg-res & $20 / 20$ \\
\hline \multicolumn{4}{|l|}{ M. tuberculosis } \\
\hline \multicolumn{4}{|l|}{$\mathrm{H} 37 \mathrm{Rv}$} \\
\hline$m c^{2} 3122$ & phAE152 & $\Delta n a d B C::$ res-hyg-res & $4 / 4$ \\
\hline $\mathrm{mc}^{2} 3123$ & phAE153 & $\Delta l e u C D:$ :res-hyg-res & $20 / 20$ \\
\hline $\mathrm{mc}^{2} 3124$ & phAE173 & $\Delta \mathrm{Rv} 3291 \mathrm{c}:$ :res-hyg-res & $5 / 5$ \\
\hline $\mathrm{mc}^{2} 6001$ & phAE187 & $\Delta$ panCD::res-byg-res & $8 / 8$ \\
\hline \multicolumn{4}{|l|}{ Erdman } \\
\hline $\mathrm{mc}^{2} 3510$ & phAE188 & $\Delta \mathrm{Rv} 0867$ : :res-hyg-res & $3 / 3$ \\
\hline $\mathrm{mc}^{2} 3511$ & phAE188 & $\Delta \mathrm{Rv} 0867:$ : res (unmarked) & \\
\hline \multicolumn{4}{|l|}{ CDC1551 } \\
\hline $\mathrm{mc}^{2} 6002$ & phAE187 & $\Delta$ panCD::res-hyg-res & $8 / 8$ \\
\hline $\mathrm{mc}^{2} 3026 \Delta l y s A$ & phAE153 & $\Delta l y s A, \Delta l e u C D:$ :res-hyg-res & $11 / 12$ \\
\hline $\mathrm{mc}^{2} 6020$ & phAE187 & $\Delta$ lys A5, $\Delta$ panCD::res-hyg-res & $4 / 4$ \\
\hline $\mathrm{mc}^{2} 3125$ & phAE152 & $\Delta l y s A 5, \Delta$ nadBC::res-hyg-res & $18 / 20$ \\
\hline \multicolumn{4}{|l|}{ M. smegmatis } \\
\hline $\mathrm{mc}^{2} 1494$ & phAE144 & $\Delta l y s A 4:$ :res-byg-res & $93 / 96$ \\
\hline $\mathrm{mc}^{2} 6009$ & phAE186 & $\Delta$ panC: :res-byg-res & $10 / 10$ \\
\hline
\end{tabular}

*Allelic exchanges are analysed either phenotypically as specific auxotrophs or by Southern analysis.

(3) Transfection of $M$. smegmatis at the permissive temperature of $30{ }^{\circ} \mathrm{C}$ to generate mycobacteriophage-packaged shuttle phasmids. This step involves the conversion of the recombinant shuttle cosmids purified from E. coli $\mathrm{Hyg}^{\mathrm{R}}$ transductants into mycobacteriophage-packaged DNA molecules. This is achieved by transfection of $M$. smegmatis cells with purified cosmids and plating for phage plaques at the permissive temperature $\left(30^{\circ} \mathrm{C}\right)$. Transfection frequencies of $10^{3}-10^{4}$ p.f.u. per $\mu \mathrm{g}$ DNA are routinely obtained. High-titre transducing lysates $\left(10^{10}-10^{11}\right.$ p.f.u. $\left.\mathrm{ml}^{-1}\right)$ can be readily obtained by propagation of the mycobacteriophage in M. smegmatis.

(4) Phage infection (transduction) of mycobacteria at the non-permissive temperature of $37^{\circ} \mathrm{C}$. In this step the AES, generated as part of the specialized transducing phage, is transferred with high efficiency into the recipient mycobacteria by transduction at the non-permissive tem- perature. Since upon infection of the recipient cells at non-permissive temperature $\left(37^{\circ} \mathrm{C}\right)$ phage replication is restricted, a large number of abortive transductants are accumulated. Allelic exchange occurs as a result of a double crossover between the homologous DNA arms flanking the disrupted gene. When plated on selective medium, antibiotic-resistant transductants are obtained with a mean frequency of $10^{-5}-10^{-7}$ per total number of cells transduced. Of these antibiotic-resistant transductants, $\sim 95 \%$ show the desired mutant phenotype when byg is used as the reporter gene.

(5) Unmarking the deletion mutation by transient expression of $\boldsymbol{\gamma} \boldsymbol{\delta}$-tnpR. For unmarking of the deletion mutations generated by specialized transduction the helper plasmid pYUB870, expressing the $\operatorname{tn} p R$ gene under the control of hsp60 promoter, was constructed. The $s a c B$ gene included in the plasmid provides negative selection for the 
Specialized transduction in mycobacteria

Table 2. Auxotroph analysis of $B C G \operatorname{Kan}^{R}$ colonies following phAE128 transduction at different outgrowth time intervals

\begin{tabular}{|c|c|c|c|c|c|c|}
\hline \multirow{2}{*}{$\begin{array}{l}\text { Outgrowth } \\
\text { time }(\mathbf{h})\end{array}$} & \multicolumn{2}{|c|}{ BCG Pasteur } & \multicolumn{2}{|c|}{ BCG Copenhagen } & \multicolumn{2}{|c|}{ BCG Moreau } \\
\hline & $\operatorname{LysA}^{-} / \operatorname{Kan}^{\mathrm{R}}$ & $\begin{array}{l}\text { Allelic exchange } \\
(\%)\end{array}$ & $\operatorname{LysA}^{-} / \operatorname{Kan}^{\mathrm{R}}$ & $\begin{array}{l}\text { Allelic exchange } \\
(\%)\end{array}$ & $\operatorname{LysA}^{-} / \operatorname{Kan}^{\mathrm{R}}$ & $\begin{array}{l}\text { Allelic exchange } \\
(\%)\end{array}$ \\
\hline 6 & $34 / 286$ & 12 & $10 / 146$ & 7 & $2 / 128$ & 2 \\
\hline 12 & $104 / 372$ & 28 & $37 / 289$ & 13 & $0 / 146$ & 0 \\
\hline 24 & $168 / 495$ & 34 & $125 / 432$ & 29 & $13 / 230$ & 6 \\
\hline
\end{tabular}

spontaneous loss of the helper plasmid when plated on media containing sucrose.

\section{Specialized transduction in $M$. bovis BCG and $M$. tuberculosis}

To test the specialized transduction system for the construction of isogenic mutant strains containing defined mutations in slow-growing mycobacteria a number of different auxotrophic mutations in several substrains of $M$. bovis BCG and M. tuberculosis were constructed (Table 1). For the construction of the lysine auxotrophs in $M$. bovis BCG three different substrains - Pasteur, Copenhagen and Moreau-were infected with the specialized transducing phage phAE128 containing the $M$. tuberculosis $\Delta l y s A 5$ allele (Pavelka \& Jacobs, 1999) marked with the res-aph-res gene cassette flanked by approximately $1 \mathrm{~kb}$ homologous DNA. Since the time required for optimal recombination in the slowgrowing mycobacteria was unknown, cell/phage mixtures were incubated at $37^{\circ} \mathrm{C}$ for different times before plating on complete medium containing kanamycin (Table 2). $\mathrm{Kan}^{\mathrm{R}}$ colonies were obtained at frequencies in the range of $5 \times 10^{-5}$ to $1 \times 10^{-6}$ of the input cells for all three BCG strains at all three incubation times. Cell titres of the control cultures, not infected with the phage, determined at the start of infection and $24 \mathrm{~h}$ later, revealed no significant change in cell numbers (data not shown). Surprisingly, no difference in the number of $\operatorname{Kan}^{\mathrm{R}}$ colonies was observed between the cells infected with the transducing phage and the control cultures not infected with the transducing phage. Therefore to test for allelic exchange events, all of the $\mathrm{Kan}^{\mathrm{R}}$ transductants were screened for lysine auxotrophy. The highest percentage of auxotrophs was observed in BCG Pasteur and BCG Copenhagen after $24 \mathrm{~h}$ outgrowth time $(34 \%$ and $29 \%$, respectively), where the percentage of the auxotrophs nearly tripled between the $6 \mathrm{~h}$ outgrowth time and the $24 \mathrm{~h}$ outgrowth time. Although an unexpectedly low frequency of allelic exchange was observed for the BCG Moreau strain, this increase in the number of auxotrophs after $24 \mathrm{~h}$ outgrowth time (from $2 \%$ to $6 \%$ ) was also observed. Analysis of the lysine auxotrophs by PCR, using locus-specific primers (10 auxotrophic clones for each strain) confirmed that the mutation was due to an allelic exchange of the wild-type allele with the $\Delta l y s A 5:$ res-aph-res allele (data not shown). The results from PCR analysis were confirmed by Southern blotting (Fig. 3). For all three BCG strains, the lysine auxotrophs had a single hybridization band, which was of the predicted size of the $\Delta l y s A 5:$ :res$a p h-r e s$ allele. In contrast, the parent strains or the $\mathrm{Kan}^{\mathrm{R}}$ prototrophic strains had a single band that corresponded to the wild-type lys $A^{+}$allele. A high proportion of spontaneous $\mathrm{Kan}^{\mathrm{R}}$ colonies was also observed in all three substrains of BCG when this gene cassette was used to genetically mark the deletion mutation in the leuCD operon (data not shown). In these experiments when $\mathrm{Kan}^{\mathrm{R}}$ transductants were screened for an auxotrophic phenotype true allelic exchange events were observed at a frequency varying between $10 \%$ and $35 \%$ of the population of $\mathrm{Kan}^{\mathrm{R}}$ transductants, depending on the number of transductants screened.

Due to the very high frequency of spontaneous kanamycin resistance observed in the slow-growing mycobacteria $\left(10^{-6}\right.$ of the input cells, which is nearly identical to the frequency of homologous recombination), in all further experiments the hygromycin-resistance gene cassette rather than the $a p h$ gene was used as the selectable marker. This is because this gene cassette was found to provide a much more stringent selection. Using this reporter gene, we constructed specialized transducing phages to engineer $\triangle$ panCD::res-hyg-res deletion mutations in M. bovis BCG Pasteur (Table 1). A transduction frequency of $10^{-6}$ of the input cells for the $\mathrm{Hyg}^{\mathrm{R}}$ marker was observed, whereas virtually no $\mathrm{Hyg}^{\mathrm{R}}$ colonies were observed when the control cells, not infected with the transducing phage, were plated on hygromycin selective media. Using this selectable marker gene we successfully engineered targeted deletions in five different chromosomal loci of $M$. tuberculosis substrains H37Rv, Erdman and CDC1551 (Table 1). In the course of constructing these mutant strains in numerous transduction experiments we consistently obtained high numbers of $\mathrm{Hyg}^{\mathrm{R}}$ transductants, ranging from experiment to experiment between $10^{-5}$ and $10^{-6}$ of the input cells (50 to more than 300 per experiment). As expected, in all cases the $\mathrm{Hyg}^{\mathrm{R}}$ transductants tested (typically 3-20 clones in each experiment) had the predicted auxotrophic phenotype. The correct structure of the deleted alleles was confirmed by PCR using locus-specific primers (data not shown) or Southern blotting (Fig. 3). 
(a)

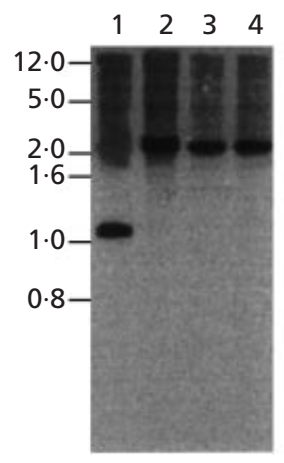

$\begin{array}{llll}5 & 6 & 7 & 8\end{array}$

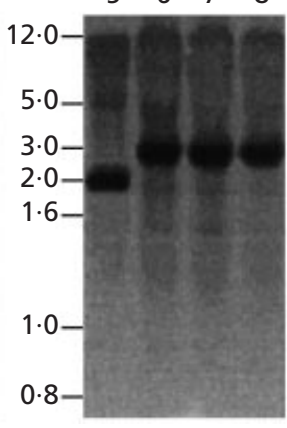

(b)

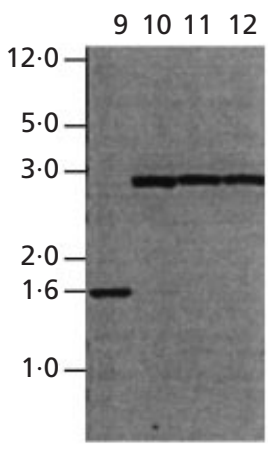

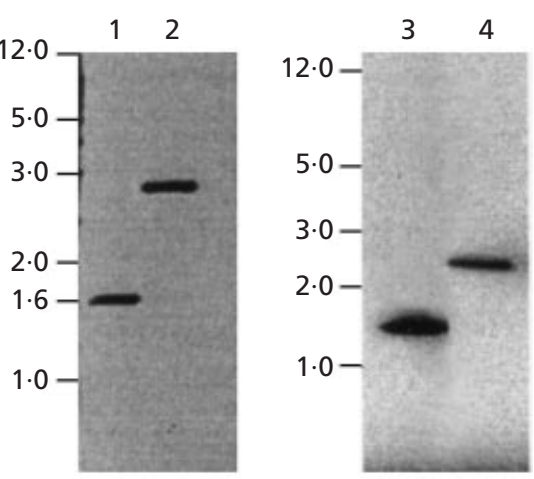

Fig. 3. A composite of Southern blot analysis of $M$. bovis BCG and $M$. tuberculosis auxotroph mutants. (a) Lanes 1-4, genomic DNA from BCG Pasteur (lane 1) and $\triangle$ lysA::res-aph-res auxotroph mutants of: BCG Pasteur (lane 2), BCG Copenhagen (lane 3) and BCG Moreau (lane 4) digested with ApaLI and probed with the lysA PCR product from BCG Pasteur wild-type genomic DNA. The wild-type fragment is the expected $1 \cdot 1 \mathrm{~kb}$, while the genomic DNA from the auxotrophic strains has the expected $2 \cdot 2 \mathrm{~kb}$ fragment. Lanes 5-8, genomic DNA from BCG Pasteur wild-type (lane 5) and $\triangle$ leuCD::res-aph-res auxotroph mutants of BCG Pasteur (lane 6), BCG Copenhagen (lane 7) and BCG Moreau (lane 8) digested with Sacll and probed with the $3.3 \mathrm{~kb}$ Pstl-HindlII fragment from pYUB804. The wild-type fragment is the expected $1.9 \mathrm{~kb}$ while the genomic DNA from the auxotrophic strains has the expected $3.4 \mathrm{~kb}$ fragment. Lanes 9-12, genomic DNA from BCG Pasteur wild-type (lane 9) and $\triangle$ nadBC::res-hyg-res auxotroph mutants of BCG Pasteur (lane 10), BCG Copenhagen (lane 11) and BCG Moreau (lane 12) digested with BsaAl and probed with the $1.7 \mathrm{~kb} \mathrm{Kpnl-SaCl}$ fragment from pYUB862. (b) Lanes 1 and 2, genomic DNA from $M$. tuberculosis H37Rv wild-type (lane 1) and the $\triangle$ nadBC::res-hyg-res auxotroph mutants (lane 2) digested with BsaAl and probed with the $1.7 \mathrm{~kb} K p n l-S a c l$ fragment from pYUB862. The wild-type fragment is the expected $1.6 \mathrm{~kb}$ while the genomic DNA from the auxotrophic strains has the expected $2.9 \mathrm{~kb}$ fragment. Lanes 3 and 4, genomic DNA from $M$. tuberculosis H37Rv wild-type (lane 3) and the $\triangle$ panCD::res-hyg-res auxotroph mutant (lane 4). Genomic DNAs were digested with BssHII, and probed with a $716 \mathrm{bp}$ downstream region flanking the panCD operon. The wild-type fragment is the expected $1.48 \mathrm{~kb}$, while the genomic DNA from the auxotrophic strain has the expected $2.65 \mathrm{~kb}$ fragment.

\section{Specialized transduction in $M$. smegmatis}

In $M$. smegmatis $\mathrm{mc}^{2} 155$ specialized transduction was used to generate $\Delta l y s A$ and $\Delta$ panC auxotrophic mutants marked with the hygromycin reporter gene. M. smegmatis cells were adsorbed with the transducing phages and phage/cell mixtures were plated on complete medium containing hygromycin. When $\mathrm{Hyg}^{\mathrm{R}}$ transductants were screened for auxotrophy, a high percentage $(95 \%$ on average) of the transductants were lysine auxotrophs. Southern analysis confirmed that the lysine-auxotrophic phenotype was due to an allelic exchange of the wild-type lys $A^{+}$allele with the mutated $\Delta$ lys A4::res-hyg-res allele (data not shown). Comparable transduction frequencies of the hygromycin resistance marker were obtained when specialized transduction was employed to generate a $\Delta$ panC deletion mutation in $M$. smegmatis. In this experiment when several representative $\mathrm{Hyg}^{\mathrm{R}}$ transductants were tested on minimal medium they all showed an auxotrophic phenotype, requiring supplementation of the media with pantothenate for optimal growth. Analysis by PCR using locus-specific primers, and Southern analysis (data not shown), confirmed the predicted configuration of the $\triangle$ pan $C$ deletion allele.

\section{Construction of unmarked deletion mutations in $\boldsymbol{M}$. tuberculosis}

One of the benefits of engineering unmarked mutations in M. tuberculosis is that multiple mutations can be generated in a single strain by using a three-step process of (1) mutagenesis (2) unmarking the deletion mutant and (3) introducing a second mutation using the original selectable marker. The specialized res-byg-res gene cassette, described above, contains the specific DNA binding sites (res) for a site-specific $\gamma \delta$ resolvase, the product of the tnpR gene of E. coli transposon

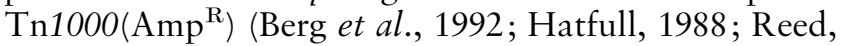
1981). Transient expression of the resolvase gene promotes site-specific recombination between the res sites. The final result is a precise excision of the hygromycinresistance gene, leaving the deletion site unmarked. We constructed a helper plasmid expressing the $\gamma \delta$ resolvase under transcriptional control of the $h s p 60$ promoter. We also included the $s a c B$ gene in this plasmid to provide a negative selection for spontaneous plasmid loss. To generate unmarked deletion mutations we used the helper plasmid pYUB870 to unmark the M. tuberculosis $\Delta \mathrm{Rv} 0867 \mathrm{c}$ : :res-hyg-res mutant strain. After transformation by electroporation with pYUB870 and plating onto medium containing kanamycin, a total of 20-30 $\mathrm{Kan}^{\mathrm{R}}$ colonies were obtained and screened by a pickand-patch method (streaking on 7H10 agar alone and on 7H10 agar with $50 \mu \mathrm{g}$ hygromycin $\mathrm{ml}^{-1}$ ) for hygromycin sensitivity. A hygromycin-sensitive clone was grown in liquid medium in the absence of antibiotic selection, and genomic DNA was prepared and tested by Southern blot analysis for loss of the hygromycin-resistance gene cassette. The analysis of one such clone is shown in Fig. 4. Southern blotting with different gene probes as well as 
(a) 1 M. tuberculosis
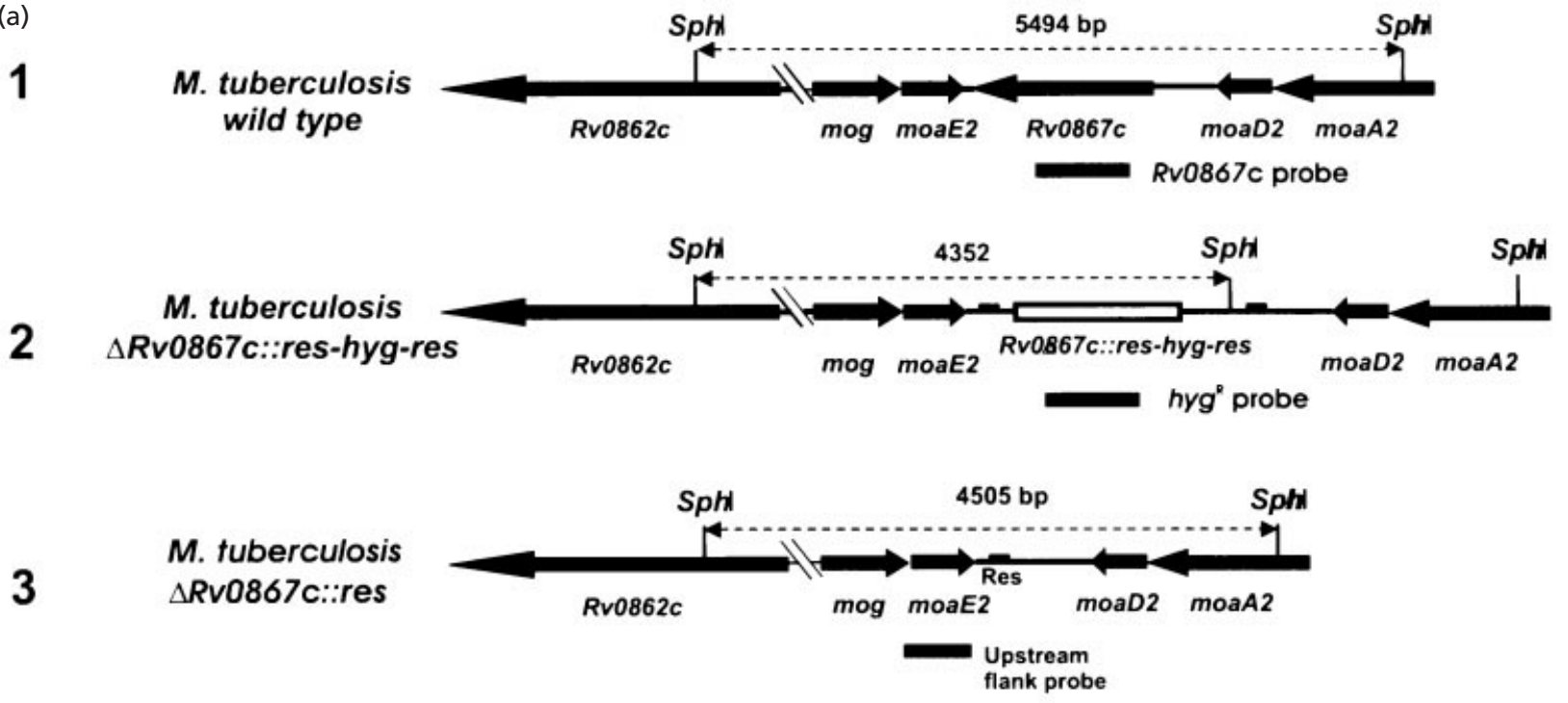

(b)

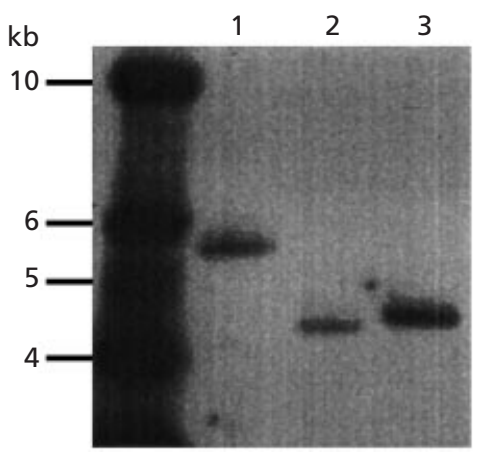

Upstream probe

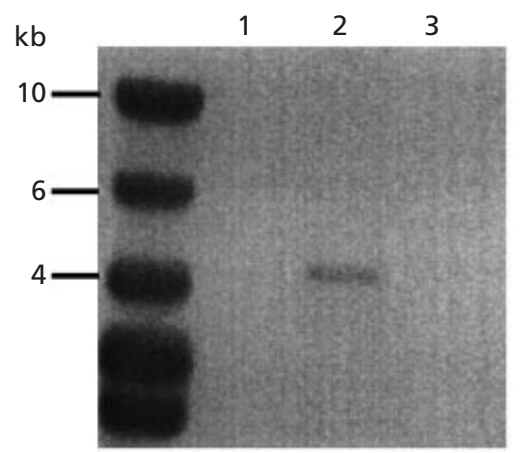

hyg $g^{\mathrm{R}}$ probe

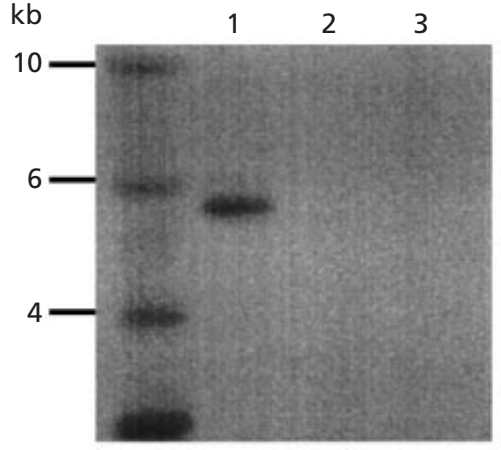

Rv0867c probe

Fig. 4. Deletion of Rv0867c from the chromosome of $M$. tuberculosis by specialized transduction, and excision of the res-hyg-res gene cassette by $\operatorname{tnp} R$ to generate an unmarked deletion strain. (a) Schematic diagram of the Rv0867c region of the chromosome of $M$. tuberculosis (Erdman). Also shown is the position of the three hybridization probes: the Rv0867c ORF; an internal portion of the hyg gene; and the upstream flanking region of the Rv0867c gene. (b) Southern blot hybridizations of Sphl-digested chromosomal DNA from $M$. tuberculosis wild-type (lanes 1), the $\Delta$ Rv0867c:: res-hyg-res strain (lanes 2) and the $\Delta$ Rv0867c-resolved strain (lanes 3). The upstream flank probe yields hybridizing bands of the predicated sizes in all three strains, while the Rv0867c gene probe hybridizes only to the wild-type strain. The hyg gene probe hybridizes only to the $\Delta$ Rv0867c::res-hyg-res strain. The Sphl restriction sites are indicated, as are the sizes of fragments hybridizing to the three probes. Deletion of Rv0867c with its replacement by the res-hyg-res cassette results in a smaller hybridizing band, since the cassette introduces an additional Sphl site. The loss of both the res-hyg-res cassette and the additional Sphl site mediated by the resolvase results in an only slightly larger hybridizing band.

with a probe specific for the hyg gene all showed that the res-byg-res cassette had been excised, giving the correct fragment sizes. This clone was then plated onto sucrosecontaining medium and single $\mathrm{Kan}^{\mathrm{S}} \mathrm{Hyg}^{\mathrm{S}}$ colonies obtained.

\section{DISCUSSION}

Several groups have demonstrated the use of suicide plasmid vectors to deliver AESs for insertional mutagenesis in both fast- and slow-growing mycobacteria (Berthet et al., 1998; Frischkorn et al., 1998; Knipfer et al., 1997; Parish \& Stoker, 2000; Pavelka \& Jacobs, 1996; Pelicic et al., 1997; Pelicic et al., 1996a, b). One of the limitations in using transformation as a means to deliver the AES for generation of targeted gene disruptions is the high frequency of illegitimate recombination in these bacterial species and the comparatively low transformation efficiency, especially in the slow-growing pathogenic mycobacteria.

Based on our previous experience in using conditionally replicating shuttle phasmid vectors (Hatfull, 1996; Hatfull et al., 1994; Jacobs et al., 1991) for efficient delivery of transposon constructs and generation of transposon libraries in both M. bovis BCG and M. tuberculosis (Bardarov et al., 1997; Cox et al., 1999) we sought the opportunity to extend their usage by 
modifying them into in vitro-generated specialized transducing phages. Specialized transduction as a method for delivery of recombinant DNA into mycobacteria has several useful features. Firstly, the specialized transduction phage system described in this study can be readily generalized to any set of genes by virtue of the facile cloning properties of the shuttle phasmids and the properties of the conditionally replicating mycobacteriophage vector. Once a specific mutated allele is engineered into the phage, that mutated allele can be introduced into any fast- or slow-growing mycobacterial species. The conditionally replicating shuttle phasmids used in this system as AES delivery vectors can be propagated in variety of ways: they can be electroporated into E. coli, where they behave as multicopy plasmids; they can be packaged in vitro into $\lambda$ phage heads and transduced in E. coli; the recombinant phasmids can be transfected into M. smegmatis, where at the permissive temperature $\left(30^{\circ} \mathrm{C}\right)$ they behave as lytic phages and high-titre transducing phage lysates could be produced; and they can be transduced with high efficiency into the mycobacterial hosts at nonpermissive temperature $\left(37^{\circ} \mathrm{C}\right)$, where they behave as non-replicating circular replicons giving rise to progeny of abortive transductants. Secondly, the specialized transducing mycobacteriophages used in this study provide a natural counterselection mechanism for the integration of the whole phage, or parts of it, into the host chromosome by single crossover or illegitimate recombination. If such an integration event occurs, expression of the phage genes, as a part of the chromosome, will be deleterious to the host cell. Thirdly, the broad host range of the mycobacteriophage TM4 allows specialized transduction to be used for efficient delivery of AESs for the generation of targeted gene disruptions into many genetically and clinically important mycobacterial species. Once a specific mutation is generated in a defined genetic background the mutant strain becomes a useful reagent for engineering of series of isogenic mutant strains that differ by that single mutated gene. Moreover, it should be rather straightforward to extend this genetic strategy to many other bacterial species for which specific phages exist.

We have demonstrated the utility and reproducibility of specialized transduction as a highly efficient method for the delivery of homologous DNA substrates for allelic exchanges in both fast- and slow-growing mycobacteria. Using the M. tuberculosis sequence database (Cole et al., 1998), seven deletion mutant alleles were engineered. When the mutant alleles were introduced by transduction into the host mycobacteria the observed recombination frequencies were in the $10^{-6}$ range; thus, these mutants could not be constructed by electroporation with a suicide plasmid, since the best electroporation efficiencies we were able to obtain with M. tuberculosis are in the $10^{-5}$ range. These results amply demonstrate the potential of using in vitro-generated specialized transducing mycobacteriophages for efficient gene replacement in mycobacteria. Ultimately, such a system should prove to be very useful for the construction and development of M. tuberculosis-based vaccine strains with numerous defined non-revertible mutations.

We also constructed a plasmid, pYUB870, for the delivery and transient expression of the $\gamma \delta$ site-specific resolvase, which acts on the directly repeated res sites flanking the antibiotic-resistance marker gene. Removal of the antibiotic marker was readily achieved by incubation of a few $\mathrm{Kan}^{\mathrm{R}}$ clones obtained after transformation with the expression plasmid and plating on sucrose-containing media. Most of the $\mathrm{Kan}^{\mathrm{S}} \mathrm{Hyg}^{\mathrm{S}}$ clones had lost both the hygromycin-resistance gene and the plasmid.

In summary we have developed a novel genetic method for a single-step, highly efficient delivery of AESs for the generation of targeted gene disruptions in both fast- and slow-growing mycobacteria. Seven different mutations have been engineered in three substrains of $M$. bovis BCG and three strains of M. tuberculosis. The present study amply demonstrates the power of specialized transduction as a natural mycobacterial genetic transfer system of AESs and generation of targeted gene disruptions that should be applicable to a wide variety of mycobacterial species.

\section{ACKNOWLEDGEMENTS}

This work was supported by grants from the National Institutes of Health AI26170, AI46690, GMG2410, AI49375 and AI28927.

\section{REFERENCES}

Aldovini, A., Husson, R. N. \& Young, R. A. (1993). The uraA locus and homologous recombination in Mycobacterium bovis BCG. J Bacteriol 175, 7282-7289.

Azad, A. K., Sirakova, T. D., Rogers, L. M. \& Kolattukudy, P. E. (1996). Targeted replacement of the mycocerosic acid synthase gene in Mycobacterium bovis BCG produces a mutant that lacks mycosides. Proc Natl Acad Sci U S A 93, 4787-4792.

Balasubramanian, V., Pavelka, M. S., Jr, Bardarov, S. S., Martin, J., Weisbrod, T. R., McAdam, R. A., Bloom, B. R. \& Jacobs, W. R., Jr (1996). Allelic exchange in Mycobacterium tuberculosis with long linear recombination substrates. J Bacteriol 178, 273-279.

Bardarov, S., Kriakov, J., Carriere, C., Yu, S., Vaamonde, C., McAdam, R. A., Bloom, B. R., Hatfull, G. F. \& Jacobs, W. R., Jr (1997). Conditionally replicating mycobacteriophages: a system for transposon delivery to Mycobacterium tuberculosis. Proc Natl Acad Sci U S A 94, 10961-10966.

Berg, C. M., Vartak, N. B., Wang, G., Xu, X., Liu, L., MacNeil, D. J., Gewain, K. M., Wiater, L. A. \& Berg, D. E. (1992). The $\mathrm{m} \gamma \delta$-1 element, a small $\gamma \delta(\operatorname{Tn} 1000)$ derivative useful for plasmid mutagenesis, allele replacement and DNA sequencing. Gene 113, 9-16.

Berthet, F. X., Lagranderie, M., Gounon, P. \& 9 other authors (1998). Attenuation of virulence by disruption of the Mycobacterium tuberculosis erp gene. Science 282, 759-762.

Boshoff, H. I. M. \& Mizrahi, V. (2000). Expression of Mycobacterium smegmatis pyrazinamidase in Mycobacterium tuberculosis confers hypersensitivity to pyrazinamide and related amides. J Bacteriol 182, 5479-5485. 
Braunstein, M., Brown, A. M., Kurtz, S. \& Jacobs, W. R., Jr (2001). Two nonredundant SecA homologues function in mycobacteria. J Bacteriol 183, 6979-6990.

Cole, S. T., Brosch, R., Parkhill, J. \& 39 other authors (1998). Deciphering the biology of Mycobacterium tuberculosis from the complete genome sequence. Nature 393, 537-544. [An erratum appears in Nature 396, 190.]

Cox, J. S., Chen, B., McNeil, M. \& Jacobs, W. R., Jr (1999). Complex lipid determines tissue-specific replication of Mycobacterium tuberculosis in mice. Nature 402, 79-83.

Fitzmaurice, A. M. \& Kolattukudy, P. E. (1998). An acyl-CoA synthase (acoas) gene adjacent to the mycocerosic acid synthase (mas) locus is necessary for mycocerosyl lipid synthesis in Mycobacterium tuberculosis var. bovis BCG. J Biol Chem 273, 8033-8039.

Fleischmann, R. D., Alland, D., Eisen, J. A. \& 23 other authors (2002). Whole genome comparison of Mycobacterium tuberculosis clinical and laboratory strains. J Bacteriol (in press).

Frischkorn, K., Sander, P., Scholz, M., Teschner, K., Prammananan, T. \& Bottger, E. C. (1998). Investigation of mycobacterial recA function: protein introns in the RecA of pathogenic mycobacteria do not affect competency for homologous recombination. Mol Microbiol 29, 1203-1214.

Glickman, M. S., Cox, J. S. \& Jacobs, W. R., Jr (2000). A novel mycolic acid cyclopropane synthetase is required for cording, persistence, and virulence of Mycobacterium tuberculosis. Mol Cell 5, 717-727.

Hatfull, G. F. (1996). The molecular genetics of Mycobacterium tuberculosis. Curr Top Microbiol Immunol 215, 29-47.

Hatfull, G. F., Salvo, J. J., Falvey, E. E., Rimphanitchayakit, V. \& Grindley, N. D. (1988). Site-Specific Recombination by the $\gamma \delta$ Resolvase. Cambridge: Cambridge University Press.

Hatfull, G. F., Barsom, L., Chang, L., Donnelly-Wu, M., Lee, M. H., Levin, M., Nesbit, C. \& Sarkis, G. J. (1994). Bacteriophages as tools for vaccine development. Dev Biol Stand 82, 43-47.

Hinds, J., Mahenthiralingam, E., Kempsell, K. E., Duncan, K., Stokes, R. W., Parish, T. \& Stoker, N. G. (1999). Enhanced gene replacement in mycobacteria. Microbiology 145, 519-527.

Jacobs, W. R., Jr, Kalpana, G. V., Cirillo, J. D., Pascopella, L., Snapper, S. B., Udani, R. A., Jones, W., Barletta, R. G. \& Bloom, B. R. (1991). Genetic systems for mycobacteria. Methods Enzymol 204, 537-555.

Kalpana, G. V., Bloom, B. R. \& Jacobs, W. R., Jr (1991). Insertional mutagenesis and illegitimate recombination in mycobacteria. Proc Natl Acad Sci US A 88, 5433-5437.

Knipfer, N., Seth, A. \& Shrader, T. E. (1997). Unmarked gene integration into the chromosome of Mycobacterium smegmatis via precise replacement of the pyrF gene. Plasmid 37, 129-140.

Lenox, E. S. (1955). Transduction of linked genetic characters of the host by bacteriophage P1. Virology 1, 190-206.

Masters, M. (1996). Generalized transduction. In Escherichia coli and Salmonella: Cellular and Molecular Biology, pp. 2421-2441. Edited by F. C. Neidhardt and others. Washington, DC: American Society for Microbiology.

McFadden, J. (1996). Recombination in mycobacteria. Mol Microbiol 21, 205-211.

Parish, T. \& Stoker, N. G. (2000). Use of a flexible cassette method to generate a double unmarked Mycobacterium tuberculosis tly A plcABC mutant by gene replacement. Microbiology 146, 19691975.
Parish, T., Gordhan, B. G., McAdam, R. A., Duncan, K., Mizrahi, V. \& Stoker, N. G. (1999). Production of mutants in amino acid biosynthesis genes of Mycobacterium tuberculosis by homologous recombination. Microbiology 145, 3497-3503.

Pavelka, M. S., Jr \& Jacobs, W. R., Jr (1996). Biosynthesis of diaminopimelate, the precursor of lysine and a component of peptidoglycan, is an essential function of Mycobacterium smegmatis. J Bacteriol 178, 6496-6507.

Pavelka, M. S., Jr \& Jacobs, W. R., Jr (1999). Comparison of the construction of unmarked deletion mutations in Mycobacterium smegmatis, Mycobacterium bovis bacillus Calmette-Guérin, and Mycobacterium tuberculosis $\mathrm{H} 37 \mathrm{Rv}$ by allelic exchange. J Bacteriol 181, 4780-4789.

Pelicic, V., Reyrat, J. M. \& Gicquel, B. (1996a). Generation of unmarked directed mutations in mycobacteria, using sucrose counter-selectable suicide vectors. Mol Microbiol 20, 919-925.

Pelicic, V., Reyrat, J. M. \& Gicquel, B. (1996b). Positive selection of allelic exchange mutants in Mycobacterium bovis BCG. FEMS Microbiol Lett 144, 161-166.

Pelicic, V., Jackson, M., Reyrat, J. M., Jacobs, W. R., Jr, Gicquel, B. \& Guilhot, C. (1997). Efficient allelic exchange and transposon mutagenesis in Mycobacterium tuberculosis. Proc Natl Acad Sci U S A 94, 10955-10960.

Raman, S., Song, T., Puyang, X., Bardarov, S., Jacobs, W. R., Jr \& Husson, R. N. (2001). The alternative sigma factor $\mathrm{SigH}$ regulates major components of oxidative and heat stress responses in Mycobacterium tuberculosis. J Bacteriol 183, 6119-6125.

Reed, R. R. (1981). Resolution of cointegrates between transposons $\gamma \delta$ and Tn 3 defines the recombination site. Proc Natl Acad Sci US A 78, 3428-3432.

Reyrat, J. M., Berthet, F. X. \& Gicquel, B. (1995). The urease locus of Mycobacterium tuberculosis and its utilization for the demonstration of allelic exchange in Mycobacterium bovis bacillus Calmette-Guérin. Proc Natl Acad Sci U S A 92, 8768-8772.

Sambrook, J., Fritsch, E. F. \& Maniatis, T. (1989). Molecular Cloning: a Laboratory Manual, 2nd edn. Cold Spring Harbor, NY: Cold Spring Harbor Laboratory.

Sander, P., Meier, A. \& Bottger, E. C. (1995). $r p s L^{+}$: a dominant selectable marker for gene replacement in mycobacteria. Mol Microbiol 16, 991-1000.

Sirakova, T. D., Thirumala, A. K., Dubey, V. S., Sprecher, H. \& Kolattukudy, P. E. (2001). The Mycobacterium tuberculosis $p k s 2$ gene encodes the synthase for the hepta- and octamethyl-branched fatty acids required for sulfolipid synthesis. J Biol Chem 276, 16833-16839.

Steyn, A. J. C., Collins, D. M., Hondalus, M. K., Jacobs, W. R., Jr, Kawakami, R. P. \& Bloom, B. R. (2002). Mycobacterium tuberculosis WhiB3 interacts with RpoV to affect host survival but is dispensable for in vivo growth. Proc Natl Acad Sci USA 99, 3147-3152.

Stover, C. K., de la Cruz, V. F., Fuerst, T. R. \& 11 other authors (1991). New use of BCG for recombinant vaccines. Nature 351, 456-460.

Sundar Raj, C. V. \& Ramakrishnan, T. (1970). Transduction in Mycobacterium smegmatis. Nature 228, 280-281.

Zinder, N. \& Lederberg, J. (1952). Genetic exchange in Salmonella. J Bacteriol 64, 679-699.

Received 27 June 2002; revised 11 July 2002; accepted 15 July 2002. 